\title{
Verbal Redundancy in Multimedia Learning: When Reading Helps Listening
}

\author{
Roxana Moreno \\ University of New Mexico
}

\author{
Richard E. Mayer \\ University of California, Santa Barbara
}

\begin{abstract}
Three studies investigated whether and under what conditions the addition of on-screen text would facilitate the learning of a narrated scientific multimedia explanation. Students were presented with an explanation about the process of lightning formation in the auditory alone (nonredundant) or auditory and visual (redundant) modalities. In Experiment 1, the effects of preceding the nonredundant or redundant explanation with a corresponding animation were examined. In Experiment 2, the effects of presenting the nonredundant or redundant explanation with a simultaneous or a preceding animation were compared. In Experiment 3, environmental sounds were added to the nonredundant or redundant explanation. Learning was measured by retention, transfer, and matching tests. Students better comprehended the explanation when the words were presented auditorily and visually rather than auditorily only, provided there was no other concurrent visual material. The overall pattern of results can be explained by a dual-processing model of working memory, which has implications for the design of multimedia instruction.
\end{abstract}

In multimedia learning, information is presented to students by a combination of different modalities or modes - such as spoken words and pictures. Mode refers to the format used to represent the lesson, such as words versus pictures (Paivio, 1986). Modality refers to the information-processing channel used by the learner to process the information, such as auditory versus visual (Penney, 1989). The purpose of this study was to investigate whether and under what conditions the addition of on-screen text would promote the comprehension of a narrated scientific explanation within multimedia learning environments. Of particular interest for the present study was how specific combinations of modes and modalities may affect students' learning of scientific explanations, such as when visual and auditory verbal materials (i.e., on-screen text and narration) are combined with visual and auditory nonverbal materials (i.e., animation and sounds; Moreno \& Mayer, 1999a, 1999b).

We defined verbal redundancy as the simultaneous presentation of text and narration with identical words. Therefore, verbal redundancy was a subclass of redundant information, in general, which also included the cases where verbal and nonverbal materials were used to represent the same information in different modes (Bobis, Sweller, \& Cooper, 1993; Chandler \& Sweller, 1991).

Roxana Moreno created the multimedia materials used in Experiments 1,2 , and 3 .

Correspondence concerning this article should be addressed to Roxana Moreno, Educational Psychology Program, 123 Simpson Hall, University of New Mexico, Albuquerque, New Mexico 87131-1246, or to Richard E. Mayer, Department of Psychology, University of California, Santa Barbara, California 93106. E-mail: moreno@unm.edu or mayer@psych.ucsb.edu

\section{Past Research on Redundancy}

In a review of modality effects in short-term memory, Penney (1989) reported a collection of memory experiments where words presented in two modalities (visual and auditory) produced enhanced memory recall in comparison to words presented in only one modality (Broadbent, 1956; Hede, 1980; Martin, 1980; Treisman \& Davies, 1973). More recently, Lewandowski and Kobus (1993) used a paradigm that combined recall and speed performance. Students made category decisions about words presented concurrently in the auditory and visual channels or in either single modality. Although there was no advantage on reaction time in the redundant condition, participants in that condition recalled more words than other participants (Lewandowski \& Kobus, 1993). Furthermore, verbal redundancy has proven to foster reading comprehension for less skilled readers (Montali \& Lewandowski, 1996).

A related finding in applied research is that response times and memory are facilitated when redundant signal information is presented simultaneously in two sensory channels rather than in one channel alone, a phenomenon known as the redundant signals effect (Kinchla, 1974). Research on the redundant signals effect is aimed at finding ways to enhance operational performance on bisensory tasks such as using sonar and radar (Lewandowski \& Kobus, 1993). For example, the benefits of redundant bisensory stimuli for a job task or learning situation include the improved detection and recognition of sonar targets (Colquhoun, 1975; Kobus et al., 1986; Lewandowski \& Kobus, 1989) and of lights and tones (Nickerson, 1973), and the improved rate of learning in bisensory redundant conditions with sea noise recordings (Halpern \& Lanz, 1974).

Finally, in the cognitive load literature, Kalyuga, Chandler, and Sweller $(1998,1999)$ have used the term redundancy effect to indicate learning situations in which "eliminating redundant ma- 
terial results in better performance than when the redundant material is included" (Kalyuga et al., 1998, p. 2). There are two important empirical findings in this set of studies. The first one is that students' learning of a self-contained diagram or graph is hurt by the addition of textual information that redundantly explains with words what the diagram or graph already represents with pictures (Chandler \& Sweller, 1991). This type of redundancy is between the verbal and nonverbal modes, and its negative effect on learning has been explained in terms of split attention. According to cognitive load theory, when learners need to split their attention between simultaneous text and graphics, it overburdens their limited working-memory capacity (Chandler \& Sweller, 1991). The second finding is that computer-based instructional materials consisting of concurrent diagrams and auditory verbal information are more efficient than those consisting of concurrent diagrams and on-screen text or concurrent diagrams and redundant auditory and visual verbal information (Kalyuga et al., 1999). This second type of redundancy between visual and auditory verbal materials has also been interpreted as resulting from the split attention of adding an unnecessary visual input to already intelligible graphic materials.

The converging findings in the theoretical and applied literatures is that bisensory redundant stimulus presentations are more efficiently processed by the human cognitive system. However, the negative effects of learning with diagrams and verbally redundant messages seem to contradict this conclusion. Therefore, one of the goals of this study was to reconcile the discrepancy between the seemingly opposing findings of cognitive load literature with the rest of the literature on verbal redundancy.

The present study extends previous research on redundancy in the following four ways: by (a) examining a verbal-redundancy effect with complex stimuli (a cause-and-effect explanation about a scientific system); (b) using multiple dependent measures of learning, including retention, transfer, and visual-verbal matching; (c) using a computer-based multimedia environment; and (d) combining verbally redundant learning situations with other nonverbal visual and auditory learning materials.

\section{Dual-Processing Systems in Working Memory}

Our research was designed to test a dual-processing theory of multimedia learning. The primary assumptions of the theory are (a) working memory includes an auditory working memory and a visual working memory (Baddeley, 1986, 1992); (b) each working memory store has a limited capacity (Sweller, 1988, 1989; Chandler \& Sweller, 1992); and (c) learning from verbal and nonverbal information occurs when relevant information in each mode is selected, organized into a coherent representation, and connected with each other (Clark \& Paivio, 1991; Moreno \& Mayer, 2000; Paivio, 1986).

In this study, we focused on the following two questions: (a) Does the addition of on-screen text to an otherwise intelligible spoken explanation promote the deep understanding of a complex scientific system? and (b) How is the processing of verbal information affected by the presentation of additional nonverbal information, such as graphics and sounds?

To answer the first question, we compared the learning outcomes of students who are presented with an auditory explanation of how the process of lightning formation works with those of students who are presented with an identical explanation plus on-screen text. To answer the second question, we examined the learning consequences of adding a sequential or simultaneous animation and environmental sounds to the explanation. To assess meaningful learning, we used multiple measures of understanding of the lightning formation process, including being able to describe the process of lightning (retention test), to give the verbal names for elements in the animation (matching test), and most importantly, to generate solutions to new problems (transfer test).

How would the presentation of redundant verbal information affect students' processing of a multimedia message? According to dual-processing theory, visually presented information is processed-at least initially_in visual working memory whereas auditorily presented information is processed-at least initially - in auditory working memory. For example, by reading text with concurrent narration, students represent the text in visual working memory and represent the corresponding narration in auditory working memory. Because the auditory and visual processing channels are independent (Penney, 1989), students can hold both representations in working memory at the same time and build referential connections between them (Mayer \& Moreno, 1998; Moreno \& Mayer, 1999a, 1999c). When narration and on-screen text are processed simultaneously, students are able to integrate both inputs and build a coherent verbal representation. Therefore, consistent with prior findings on verbal redundancy (Lewandowski \& Kobus, 1993), our dual-processing model of multimedia learning predicts enhanced learning from redundant messages.

\section{Experiment 1}

In this study, some students received a verbal explanation about the process of lightning formation in the auditory modality as narration (Group $\mathrm{N}$ ) or in both the auditory and visual modalities as narration and on-screen text (Group NT). In addition, other students viewed a portion of an animation that depicted the process of lightning, preceding the corresponding narration segment (Group A-N) or preceding the corresponding narration and onscreen text segment (Group A-NT). By making the presentation sequential, we intended to avoid confounding the effects of redundancy with the effects of split attention (Chandler \& Sweller, 1992; Mayer \& Moreno, 1998; Moreno \& Mayer, 1999a). According to the dual-processing hypothesis, Groups NT and A-NT should outperform Groups $\mathrm{N}$ and $\mathrm{A}-\mathrm{N}$, respectively, on retention, transfer, and matching tests. In addition, as the visual information contained in the animation provides an extra code to represent the scientific system and associate with the explanation, it was predicted that Groups A-NT and A-N would outperform Groups NT and N. This prediction is consistent with past research in multimedia learning where learners understand explanations better when they receive words and corresponding pictures rather than words alone (Mayer, 1997).

\section{Method}

Participants and design. The participants were 74 college students recruited from the psychology subject pool at the University of California, Santa Barbara. Ages ranged from 18 to 26 ( $M=18.90$ years). Nineteen participants served in the $\mathrm{N}$ group ( $79 \%$ females, $21 \%$ males), 19 in the NT 
group (68\% females, $32 \%$ males), 18 in the A-N group ( $74 \%$ females, $26 \%$ males), and 18 in the A-NT group ( $88 \%$ females, $12 \%$ males).

Materials and apparatus. For each participant, the paper-and-pencil materials consisted of a participant questionnaire, a retention test, a matching test, and a four-page transfer test, with each typed on 8.5 by 11-in. sheets of paper. The participant questionnaire solicited information that concerned the participant's SAT scores, gender, and meteorology knowledge. Meteorology knowledge was assessed by using a seven-item knowledge checklist and a five-item self-rating. The checklist consisted of instructions to "please place a check mark next to the items that apply to you," following a list of six items: (a) "I regularly read the weather maps in the newspaper." (b) "I know what a cold front is." (c) "I can distinguish between cumulous and nimbus clouds." (d) "I know what a low pressure system is." (e) "I can explain what makes the wind blow." (f) "I know what this symbol means: [symbol for cold front]." and (g) "I know what this symbol means: [symbol for warm front]." The five-item self-rating asked the participants to rate their knowledge of weather by placing a check mark next to very little, between very little and average, average, between average and very much, and very much.

The retention test contained the following instructions at the top of the sheet: "Please write down an explanation of how lightning works." The matching test presented four frames from the animation along with the following instructions:

Circle cool moist air and write $\mathrm{C}$ next to it. Circle the warmer surface and write W next to it. Circle the updraft and write U next to it. Circle the freezing level and write F next to it. Circle the downdraft and write D next to it. Circle the gusts of cool wind and write G next to it. Circle the stepped leader and write $\mathrm{S}$ next to it. Circle the return stroke and write $\mathrm{R}$ next to it.

The transfer test consisted of the following four questions, each typed on a separate sheet: (a) "What could you do to decrease the intensity of lightning?" (b) "Suppose you see clouds in the sky, but no lightning. Why not?" (c) "What does air temperature have to do with lightning?" and (d) "What causes lightning?"

The computerized materials consisted of four computer programs for multimedia presentations on how the lightning process works. In the A-N and A-NT versions, an animation depicted air moving from the ocean to the land, water vapor condensing to form a cloud, the rising of the cloud beyond the freezing level, the formation of crystals in the cloud, the movement of updrafts and downdrafts, the building of electrical charges within the cloud, the division of positive and negative charges, the traveling of a negative stepped leader from the cloud to the ground, the traveling of a positive stepped leader from the ground to the cloud, the negative charges following the path to the ground, the meeting of the negative leader with the positive leader, and the positive charges following the path towards the cloud. The A-N version consisted of 16 successive segments of the animation followed by a corresponding segment of the narration. The A-NT version consisted of 16 successive cycles of animation followed by a corresponding segment of the narration and on-screen text, which contained identical words. The computerized materials were adapted from those used in previous studies (Mayer \& Moreno, 1998; Moreno \& Mayer, 1999a). The N and NT versions were identical to the A-N and A-NT versions, respectively, with the exception that the animation had been deleted throughout the programs so there were temporal pauses between segments. All programs had a total duration of $300 \mathrm{~s}$. The multimedia animations were developed by using Director 5 and Soundedit 16 (Macromedia, 1997).

The apparatus consisted of five Macintosh IIci computer systems, which included 14-in. monitors and Sony headphones.

Procedure. Participants were tested in groups of one to five per session. Each participant was randomly assigned to a treatment group (N, NT, $\mathrm{A}-\mathrm{N}$, or A-NT) and was seated at an individual cubicle in front of a computer.
First, participants completed the participant questionnaire at their own rates. Second, the experimenter presented oral instructions, stating that the computer would present an explanation of how the process of lightning works and that when the computer was finished, the experimenter would have some questions for the participants to answer. All participants were told to put on headphones and to press the space bar to begin the presentation. Third, the respective version of the explanation was presented once to all participants on pressing the space bar. Fourth, when the presentation was finished, the experimenter presented oral instructions for the test, stating that there would be a series of question sheets and that for each sheet, the participant should continue to work until told to stop. Fifth, the retention test was distributed along with instructions to write down an explanation of how lightning works. After $5 \mathrm{~min}$, the test sheet was collected. Then the transfer test sheets were presented one at a time for 3 min each, with each sheet collected by the experimenter before the subsequent sheet was handed out. Then, the matching test was presented and collected after $3 \mathrm{~min}$.

\section{Results and Discussion}

Table 1 summarizes the mean scores (and standard deviations) for the four groups on each of the three tests in Experiment 1. For each of the three dependent measures (i.e., retention, transfer, and matching) the data were subjected to a two-way analysis of variance (ANOVA) with the between-subjects factors being redundant or nonredundant verbal information (NT and A-NT vs. N and A-N, respectively) and presence or absence of animation (A-N and A-NT vs. N and NT, respectively).

Scoring. A scorer who was not aware of the treatment condition of each participant determined the retention score, matching score, and transfer score for each participant. A retention score was computed for each participant by counting the number of major idea units (out of 19 possible) that the participant produced on the retention test. One point was given for correctly stating each of the following 19 idea units regardless of wording: (a) cool air moves, (b) it becomes heated, (c) it rises, (d) water condenses, (e) the cloud extends beyond the freezing level, (f) crystals form, (g) water and crystals fall, (h) it produces updrafts and downdrafts, (i)

Table 1

Mean Retention, Transfer, and Matching Scores and Corresponding Standard Deviations for Four Groups (Experiment 1)

\begin{tabular}{|c|c|c|c|c|c|c|}
\hline \multirow[b]{3}{*}{ Group } & \multicolumn{6}{|c|}{ Dependent measure } \\
\hline & \multicolumn{2}{|c|}{ Retention } & \multicolumn{2}{|c|}{ Transfer } & \multicolumn{2}{|c|}{ Matching } \\
\hline & $M$ & $S D$ & $M$ & $S D$ & $M$ & $S D$ \\
\hline $\mathrm{N}$ & 5.21 & 2.78 & 0.86 & 0.94 & 4.63 & 2.09 \\
\hline NT & 8.05 & 3.76 & 2.47 & 1.43 & 5.84 & 1.77 \\
\hline A-N & 8.72 & 3.48 & 1.33 & 1.14 & 6.83 & 1.34 \\
\hline A-NT & 11.44 & 3.62 & 3.17 & 1.30 & 7.17 & 1.47 \\
\hline Redundant & 9.70 & 4.03 & 2.81 & 1.39 & 6.49 & 1.74 \\
\hline Nonredundant & 6.92 & 3.57 & 1.11 & 1.05 & 5.70 & 2.07 \\
\hline Animation & 10.08 & 3.76 & 2.25 & 1.52 & 7.00 & 1.39 \\
\hline No animation & 6.63 & 3.57 & 1.68 & 1.44 & 5.24 & 2.01 \\
\hline
\end{tabular}

Note. The potential range of scores was 0 to 19 for the retention test, 0 to 6 for the transfer test, and 0 to 8 for the matching score. $\mathrm{N}=$ narration alone; $\mathrm{NT}=$ simultaneous narration and text; $\mathrm{A}-\mathrm{N}=$ animation followed by narration; A-NT = animation followed by narration and text. 
people feel the gusts of cool wind before the rain, (j) electrical charges build, (k) negative charges fall to the bottom of the cloud (or positive charges go to the top), (1) a step leader travels down, (m) in a step fashion, (n) the leaders meet, (o) at 165 feet from the ground, (p) negative charges rush down, (q) they produce a light that is not very bright, (r) positive charges rush up, (s) this produces the bright light people see as a flash of lightning.

A matching score was computed for each participant by counting the number of correctly labeled elements (out of eight possible) on the matching test. Participants received 1 point for each part that was circled and labeled with the appropriate letter.

A transfer score was computed for each participant by counting the number of acceptable answers that the participant produced across the four transfer problems. For example, acceptable answers for the first question about how to decrease lightning intensity included removing positive ions from the ground; acceptable answers for the second question about why there could be clouds but no lightning, included, stating that the tops of the clouds might not be high enough to freeze; acceptable answers for the third question about how temperature is related to lightning included stating that the air must be cooler than the ground; acceptable answers for the fourth question about what causes lightning included the difference in electrical charges in the cloud. Participants received no more than 3 points per problem.

Because previous studies have demonstrated that some instructional effects were stronger for low-experience learners than for high-experience learners (Mayer \& Gallini, 1990; Mayer \& Sims, 1994), we included only low-experience students in our study. We computed an experience score by tallying the number of domainrelated activities that the participant checked on the activity checklist and adding that number to the level of experience the participant checked on the five-level self-assessment (with 0 [very little], 1 [less than average], 2 [average], 3 [more than average], and 4 [very much]). We eliminated the data for any student who scored above 5 points and replaced that data with the data of a new student. Using this procedure, we replaced 5 students in Experiment 1.

Did redundant verbal explanations promote better performance on the retention, transfer, and matching tests than nonredundant explanations? The primary issue addressed in this study concerns whether students learn the presented material more deeply when the explanations are presented to them in both visual and auditory modalities. Students remembered significantly more, $F(1$, $70)=12.18, M S E=143.09, p<.01$; they generated significantly more conceptual creative solutions on the transfer test, $F(1$, $70)=36.56, M S E=53.81, p<.01$; and they correctly matched more items, $F(1,70)=3.82, M S E=11.02, p=.05$, when the verbal material was redundant than when it was not.

Did students understand explanations better when they received words and corresponding pictures rather than words alone? Students remembered significantly more when the verbal material included a relevant animation than when it did not, $F(1,70)=$ $18.75, M S E=220.26, p<.01$; they generated significantly more conceptual creative solutions on the transfer test when the animation was included than when it was not, $F(1,70)=4.02$, $M S E=5.92, p=.05$; and they correctly matched more items when the animation was included than when it was not, $F(1$, 70) $=19.92, M S E=57.47, p<.01$.
There was no significant interaction between redundancy and inclusion of visual materials for the retention, transfer, or matching scores, $F(1,70)=0.01, M S E=0.07, n s ; F(1,70)=0.20$, $M S E=0.30, n s ; F(1,70)=1.23, M S E=3.56, n s$, respectively.

Overall, verbal redundancy and animation effects were obtained on the retention, matching, and transfer tests. On each test, the redundancy effect sizes were 0.78 for the retention test, 1.62 for the transfer test, and 0.39 for the matching test.

The findings are consistent with the predictions of the dualprocessing model. Unlike previous research on redundancy effects (Kalyuga et al., 1998, 1999), adding on-screen text to a spoken explanation helped students' retention and building of a mental model of the scientific system. In the studies by Kalyuga et al. (1998, 1999), corresponding narration and printed text were presented at the same time as graphics, requiring learners to split their visual attention between the printed words and the pictorial presentation. However, split attention was eliminated in the present study by presenting the graphics before the words (or not at all). In this case, redundancy of verbal messages aided learning. Moreover, consistent with past findings on multimedia effects (Mayer, 1997), adding nonverbal visual information to scientific explanations helped students' learning by providing an extra code to be represented, associated, and integrated with the verbal code. Although visual working memory is limited in capacity, presenting the animation before the on-screen text limits the overload that may result from forcing students to split their attention between two visual inputs.

\section{Experiment 2}

How does the presentation of nonverbal visual materials affect students' understanding of a redundant explanation? In Experiment 1 , we demonstrated the beneficial effects of presenting redundant explanations when no other visual information was presented or when the explanation was preceded by corresponding chunks of an animation. However, the most common multimedia learning scenarios include explanations with simultaneous visual materials (such as animations, video, or graphics). The simultaneous presentation of narration and animations has been shown to be more effective in fostering student understanding than the simultaneous presentation of on-screen text and animations (Mayer \& Moreno, 1998; Moreno \& Mayer, 1999a). The beneficial effects of presenting narrated animations extend to sequential presentations when the segments are short, such as in this study (Moreno \& Mayer, 1999a).

Given the limited resources students have for visual information processing, using a visual modality to present both pictorial and verbal information can create an overload situation for the learner. Therefore, and similar to the negative redundancy effects found by Kalyuga et al. (1999), dual-processing theory predicts a splitattention effect (Chandler \& Sweller, 1992; Mayer \& Moreno, 1998; Moreno \& Mayer, 1999a, 1999c) in which students who learn with redundant explanations and simultaneous animations will perform more poorly on retention, transfer, and matching tests than those who receive spoken explanations alone.

The purpose of Experiment 2 was to examine the effects of presenting redundant verbal information when the learner needs to process a corresponding animation simultaneously. Students received an animation and concurrent explanation about the forma- 
tion of lightning through narration (Group AN) or through narration and on-screen text (Group ANT), or they received an animation preceding the explanation through narration (Group A-N) or through narration and on-screen text (Group A-NT).

In this experiment, we examined two effects. First, a splitattention effect was measured by comparing the groups that received concurrent presentations (AN and ANT) with the ones that received sequential presentations (A-N and A-NT). Second, a verbal-redundancy effect was measured by comparing the groups that received auditory verbal information alone (AN and A-N) with the ones that received auditory and visual verbal information (ANT and A-NT).

\section{Method}

Participants and design. The participants were 69 college students recruited from the psychology subject pool at the University of California, Santa Barbara. Ages ranged from 17 to 23 ( $M=18.80$ years). Eighteen participants served in the A-NT group (56\% females, $44 \%$ males), 18 in the ANT group (67\% females, $33 \%$ males), 17 in the A-N group (71\% females, $29 \%$ males), and 16 in the AN group (63\% females, 37\% males). Using the same procedure as in Experiment 1, we replaced 8 students who indicated that they possessed high knowledge in meteorology.

Materials and apparatus. For each participant, the paper-and-pencil materials and apparatus were identical to those used in Experiment 1. The computerized materials consisted of four computer programs on how the lightning process works, using the same words as in Experiment 1. The A-N and A-NT versions were identical to the respective A-N and A-NT versions used in Experiment 1. The AN and ANT versions were identical to the respective A-N and A-NT versions with the exception that the verbal information was presented during the relevant part of the animation rather than afterward. The multimedia programs were developed by using Director 5 and Soundedit 16 (Macromedia, 1997).

Procedure. The procedure was identical to that used in Experiment 1.

\section{Results and Discussion}

Table 2 summarizes the mean scores (and standard deviations) for the four groups on each of the three tests in Experiment 2. For each of the three dependent measures (i.e., retention, transfer, and matching) the data were subjected to a two-way ANOVA with the between-subjects factors being redundant or nonredundant verbal information (A-NT and ANT vs. A-N and AN, respectively) and simultaneous or sequential presentation order (AN and ANT vs. A-N and A-NT, respectively).

Scoring. The retention score, matching score, and transfer score for each participant were computed identically to the way they were computed in Experiment 1.

Did redundant verbal explanations promote better performance on the retention, transfer, and matching tests than nonredundant explanations? Students did not remember significantly more, $F(1,65)=0.95, M S E=7.62, n s$; they did not generate significantly more conceptual creative solutions, $F(1,65)=0.02$, $M S E=0.03, n s$; and they did not correctly match more items, $F(1$, $65)=1.07, M S E=1.35, n s$, when the verbal material was redundant than when it was not.

Did sequential or simultaneous presentations of words and animations promote students' understanding better? There were no significant differences in the retention and matching scores of students who were presented with simultaneous and sequential presentations, $F(1,65)=0.03, M S E=0.25, n s ; F(1,65)=0.04$,
Table 2

Mean Retention, Transfer, and Matching Scores and Corresponding Standard Deviations for Four Groups (Experiment 2)

\begin{tabular}{|c|c|c|c|c|c|c|}
\hline \multirow[b]{3}{*}{ Group } & \multicolumn{6}{|c|}{ Dependent measure } \\
\hline & \multicolumn{2}{|c|}{ Retention } & \multicolumn{2}{|c|}{ Transfer } & \multicolumn{2}{|c|}{ Matching } \\
\hline & $M$ & $S D$ & $M$ & $S D$ & $M$ & $S D$ \\
\hline AN & 11.06 & 2.62 & 2.06 & 1.06 & 7.50 & 0.89 \\
\hline ANT & 8.28 & 2.08 & 1.28 & 1.13 & 6.83 & 1.25 \\
\hline A-N & 8.82 & 3.15 & 2.24 & 1.30 & 7.06 & 1.20 \\
\hline A-NT & 10.28 & 3.32 & 2.94 & 1.16 & 7.17 & 1.10 \\
\hline Redundant & 9.28 & 2.91 & 2.11 & 1.41 & 7.00 & 1.17 \\
\hline Nonredundant & 9.91 & 3.08 & 2.15 & 1.18 & 7.27 & 1.07 \\
\hline Simultaneous & 9.59 & 2.71 & 1.65 & 1.15 & 7.15 & 1.13 \\
\hline Sequential & 9.57 & 3.28 & 2.60 & 1.27 & 7.11 & 1.13 \\
\hline
\end{tabular}

Note. The potential range of scores was 0 to 19 for the retention test, 0 to 6 for the transfer test, and 0 to 8 for the matching score. AN = simultaneous animation and narration; ANT $=$ simultaneous animation, narration, and text; A-N = animation followed by narration; A-NT = animation followed by narration and text.

$M S E=0.05, n s$, respectively. However, students who received sequential presentations generated more conceptual creative solutions on the transfer test than those who received simultaneous presentations, $F(1,65)=10.69, M S E=14.56, p<.01$.

A significant interaction between redundancy and presentation order for the retention and transfer scores was found, $F(1$, $65)=9.62, M S E=77.31, p<.01$, and $F(1,65)=7.05$, $M S E=9.60, p=.05$, respectively. Consistent with the predictions of a dual-processing theory of multimedia learning, students presented with redundant verbal materials outperformed students who learned with nonredundant verbal materials when the presentations are sequential. For simultaneous presentations of animations and explanations, the opposite was true: A split-attention effect between the on-screen text and the animation occurs in which the redundant message hurts rather than helps students' learning. Unlike the case of retention and transfer, the matching scores of the groups did not differ significantly from each other, $F(1$, $65)=2.05, M S E=2.58, n s$. The discrepancy in the interaction results might be because the matching test was not sensitive enough to measure students' learning processes in these presentation conditions. All students scored high on this particular measure (i.e., there was a ceiling effect; see Table 2, column 3). On each test, the redundancy effect sizes were 0.20 for the retention test, .34 for the transfer test, and .24 for the matching test.

Overall, the interaction between verbal redundancy and presentation order is consistent with a dual-processing theory of multimedia learning. Redundant verbal messages helped students' retention and problem solving transfer when dynamic graphics were not presented concurrently with on-screen text. When learners were forced to concurrently hold words and pictures in visual working memory, they were less able to devote attentional resources to building connections between them (Chandler \& Sweller, 1992; Sweller, 1988, 1989). On the other hand, when learners were presented with segments of animation followed by a corresponding explanation, relevant information in each mode could be selected, organized into a coherent representation, and 
connected with each other (Clark \& Paivio, 1991; Moreno \& Mayer, 2000; Paivio, 1986). The findings of Experiment 2 are consistent with past research on verbal redundancy and splitattention effects. First, they are congruent with prior verbal redundancy effects on memory and comprehension: Words presented in both the visual and auditory modalities enhance learning as compared to words presented in only one modality (Lewandowski \& Kobus, 1993; Montali \& Lewandowski, 1996). Second, they are congruent with past research on split-attention effects: Computerbased instructional materials that consist of concurrent diagrams and auditory verbal information are more efficient than those that consists of concurrent diagrams and redundant auditory and visual verbal information (Kalyuga et al., 1999).

\section{Experiment 3}

How does the presentation of nonverbal auditory materials affect students' understanding of a redundant explanation? According to our dual-processing model of multimedia learning, when text and narration are presented with concurrent sounds, students need to hold the auditory verbal and nonverbal sources of information in auditory working memory (Moreno \& Mayer, 2000). Although the auditory channel is limited in capacity (Baddeley, 1992), the processing of words and sounds can be represented in separate verbal and nonverbal systems and linked to each other in an associative network (Clark \& Paivio, 1991; Paivio, 1986). Unlike the case of concurrent verbal and nonverbal visual information-where attending to on-screen text is done at the expense of the learner missing part of the animation and viceversa-for the auditory channel, attending to the spoken words does not prevent listening to background sounds and vice-versa. This is particularly true for the materials used in the present study, where the environmental sounds used during the presentation did not mask or make the narrative perceptually less discernible (Moreno \& Mayer, 2000). Therefore, students who received redundant explanations were not affected by the inclusion of relevant environmental sounds. Moreover, because the combined associative strength of verbal and nonverbal representations enhanced learning, dual-processing theory predicted that students presented with environmental sounds would outperform those presented solely with verbal information.

The purpose of Experiment 3 was to determine whether the verbal-redundancy effect obtained in Experiment 1 with presentations that included nonverbal visual information would extend to the case of multimedia presentations that included nonverbal auditory information. Similar to Experiment 1, students listened to an explanation about the process of lightning formation with or without concurrent on-screen text (Groups NT and N, respectively). Additionally, for some students, we added background relevant environmental sounds (Groups NST and NS, respectively).

\section{Method}

Participants and design. The participants were 71 college students recruited from the psychology subject pool at the University of California, Santa Barbara. Ages ranged from 17 to 23 ( $M=18.78$ years). Nineteen participants served in the NST group (63\% females, $37 \%$ males), 17 in the $\mathrm{N}$ group (71\% females, $29 \%$ males), 17 in the NS group (67\% females, $33 \%$ males), and 18 in the NT group (61\% females, $49 \%$ males). Using the same procedure as in Experiment 1, we replaced 4 students who indicated that they possessed high knowledge in meteorology.

Materials and apparatus. For each participant, the paper-and-pencil materials and apparatus were identical to those used in Experiment 1. They consisted of a participant questionnaire, a retention test, a matching test, and a four-page transfer test.

The computerized materials were four programs on how the lightning process works, using the same words as in Experiment 1. The N and NT versions were identical to the $\mathrm{N}$ and NT versions for Experiment 1, respectively. The NS and NST versions were identical to the respective N and NT versions with the exception that there was a relevant set of environmental sounds during the respective portion of the explanation. The sounds were (a) a gentle wind, for the portion of the air moving from the ocean to the land; (b) water condensing in a pot, for the portion of water vapor forming a cloud; (c) a clinking sound, for the portion of the formation of crystals in the cloud; (d) a strong wind, for the portion of the downdrafts; (e) a static sound, for the portion of the building of electrical charges; (f) a crackling sound, for the portion of the traveling of the charges from the cloud to the ground and vice versa; and $(\mathrm{g})$ thunder, for the portion of the flash of lightning. The multimedia programs were developed by using Director 5 and Soundedit 16 (Macromedia, 1997).

Procedure. The procedure was identical to that used in Experiment 1.

\section{Results and Discussion}

Table 3 contains the mean scores (and standard deviations) for the four groups on each of the three tests in Experiment 3. For each of the three dependent measures (i.e., retention, transfer, and matching) the data were subjected to a two-way ANOVA with the between-subjects factors being redundant or nonredundant verbal information (NT and NST vs. N and NS, respectively) and presence or absence of sounds (NS and NST vs. N and NT, respectively).

Scoring. The retention score, matching score, and transfer score for each participant were computed identically to the way they were computed in Experiment 1.

Did redundant verbal explanations promote better performance on the retention, transfer, and matching tests than nonredundant

Table 3

Mean Retention, Transfer, and Matching Scores and Corresponding Standard Deviations for

Four Groups (Experiment 3)

\begin{tabular}{|c|c|c|c|c|c|c|}
\hline \multirow[b]{3}{*}{ Group } & \multicolumn{6}{|c|}{ Dependent measure } \\
\hline & \multicolumn{2}{|c|}{ Retention } & \multicolumn{2}{|c|}{ Transfer } & \multicolumn{2}{|c|}{ Matching } \\
\hline & $M$ & $S D$ & $M$ & $S D$ & $M$ & $S D$ \\
\hline $\mathrm{N}$ & 3.71 & 2.02 & 0.77 & 0.83 & 4.53 & 2.07 \\
\hline NT & 8.67 & 2.97 & 2.89 & 1.64 & 6.22 & 1.83 \\
\hline NS & 4.35 & 2.89 & 0.59 & 0.62 & 4.35 & 2.34 \\
\hline NST & 8.84 & 2.83 & 3.00 & 1.37 & 5.58 & 1.39 \\
\hline Redundant & 8.76 & 2.86 & 2.95 & 1.49 & 5.89 & 1.63 \\
\hline Nonredundant & 4.03 & 2.48 & 0.68 & 0.73 & 4.41 & 2.18 \\
\hline Sounds & 6.72 & 3.62 & 1.86 & 1.62 & 5.00 & 1.97 \\
\hline No sounds & 6.26 & 3.56 & 1.86 & 1.68 & 5.40 & 2.10 \\
\hline
\end{tabular}

Note. The potential range of scores was 0 to 19 for the retention test, 0 to 6 for the transfer test, and 0 to 8 for the matching score. $\mathrm{N}=$ narration alone; $\mathrm{NT}=$ simultaneous narration and text; $\mathrm{NS}=$ simultaneous narration and sounds; NST $=$ simultaneous narration, sounds, and text. 
explanations? As in Experiment 1, the primary issue addressed in this study was whether students learned an explanation more deeply when it was presented to them in both visual and auditory modalities. Students remembered significantly more when the verbal material was redundant than when it was not, $F(1$, $67)=53.64, M S E=395.43, p<.01$; they generated significantly more conceptual creative solutions on the transfer test when the verbal material was redundant than when it was not, $F(1$, $67)=62.96, M S E=91.11, p<.01$; and they correctly matched more items when the verbal material was redundant than when it was not, $F(1,67)=10.20, M S E=37.73, p<.01$.

Did students understand explanations better when they received words and corresponding environmental sounds rather than words alone? Students who were presented with environmental sounds did not remember more, $F(1,67)=0.41, M S E=3.00, n s$; they did not generate more conceptual creative solutions on the transfer test, $F(1,67)=0.01, M S E=0.02, n s$; and they did not match more items, $F(1,67)=0.80, M S E=2.98, n s$, than students who were not presented with sounds.

Similar to the case of Experiment 1, there was no significant interaction between redundancy and inclusion of sounds for the retention, transfer, or matching scores, $F(1,67)=0.13$, $M S E=0.99, n s ; F(1,67)=0.25, M S E=0.37, n s ; F(1$, $67)=0.26, M S E=0.97, n s$, respectively.

The redundancy effects obtained on the retention, transfer, and matching tests replicate those obtained in Experiment 1. The effect sizes were 1.91 for the retention test, 3.11 for the transfer test, and 0.68 for the matching test.

These results confirm the predictions of the dual-processing hypothesis for redundant verbal information. Adding on-screen text to a spoken explanation helps students build a mental model of the scientific system. However, adding relevant and coordinated auditory material — in the form of environmental sounds — did not help students understand the lightning process. The failure to find a beneficial effect for the inclusion of environmental sounds is consistent with a prior study on multimedia learning with animations, sounds, and music (Moreno \& Mayer, 2000). In this study, students were shown a narrated animation about the process of lightning with background music, sounds, both, or neither (Moreno \& Mayer, 2000, Experiment 1). On tests of retention, transfer, and verbal-visual matching, groups receiving sounds performed equivalently than groups not receiving sounds. Taken together, the results from Experiment 3 and Moreno and Mayer's study (2000, Experiment 1) suggest that the auditory information contained in the environmental sounds did not provide a strong enough cue to help students' learning. However, it is important to note that despite auditory working-memory limitations (Baddeley, 1992), the addition of sounds did not interfere with the processing of the narration. We interpret this finding as supporting a dual-processing hypothesis by failing to demonstrate overload of auditory working memory when verbal and nonverbal auditory inputs are coordinated simultaneously.

\section{General Discussion}

\section{Theoretical Implications}

We provided here an empirical test of a dual-processing theory of working memory within the domain of multimedia learning.
According to a dual-processing theory of working memory, and consistent with past research on verbal redundancy in short-term memory (Penney, 1989), students learn better with redundant rather than with nonredundant verbal explanations provided no other competing visual materials are presented simultaneously. First, when words are both presented visually and aurally, learners are able to select both pieces of information with no cognitive overload: Because visual working memory and auditory working memory work as independent processors, additional processing capacity is made available to the student when two modalities are used (Moreno \& Mayer, 1999a).

Second, when corresponding chunks of animation precede a redundant explanation, the beneficial learning effects of redundant explanations remains: Although on-screen text and pictures are processed in the visual channel, the sequential nature of the presentation does not overload visual working memory (Moreno \& Mayer, 1999a, 1999c). In Experiment 1, we demonstrated a verbalredundancy effect on retention, transfer, and matching. In addition, the findings of Experiment 1 are also consistent with dualprocessing theory: There was a multimedia effect for retention, transfer, and matching. Students learn better when they are presented with words and pictures rather than with pictures alone (Mayer, 1997).

The second goal of our research was to reconcile the seemingly opposing findings of prior verbal redundancy studies (Penney, 1989; Lewandowski \& Kobus, 1993) and cognitive load studies (Kalyuga et al., 1999). In previous research on verbal redundancy, bisensory redundant stimulus presentations were processed more efficiently than nonredundant presentations (Penney, 1989; Lewandowski \& Kobus, 1993). Conversely, Kalyuga et al. (1999) found that computer-based instructional materials, consisting of concurrent diagrams and auditory-verbal information alone, are more efficient than those consisting of concurrent diagrams and redundant auditory and visual-verbal information (Kalyuga et al., 1999). In Experiment 2, we tested the hypothesis that the verbal redundancy impairment found in the study conducted by Kalyuga et al. (1999) was due to a split-attention effect (Chandler \& Sweller, 1992; Sweller, 1988, 1989). According to dual-processing theory of working memory, when pictures and words are both presented visually (i.e., a split-attention situation), learners are able to select fewer pieces of relevant information because visual working memory is overloaded, hence learning is impaired (Mayer \& Moreno, 1998; Moreno \& Mayer, 1999a; Mousavi, Low, \& Sweller, 1995).

In Experiment 2, the disadvantage of redundant verbal explanations over spoken explanations disappeared when presentations were made sequential. When students were able to hold the graphic information before attending to the textual information in visual working memory, redundant verbal explanations enhanced learning (Moreno \& Mayer, 1999a). Thus, two seemingly contradicting findings (those from verbal redundancy research and cognitive load research) were reconciled.

Finally, in Experiment 3 we tested an additional hypothesis consistent with dual-code theory: The addition of relevant sounds to a redundant explanation will not overload auditory working memory (Paivio, 1986). The nonverbal code provided by environmental sounds acts as an extra cue stored by the learner with additional associations and retrieval paths (Anderson, 1983; Paivio, 1986). Consistent with this hypothesis, in Experiment 3, 
we demonstrated a verbal-redundancy effect for retention, transfer, and matching tests. Unlike the case for Experiment 1, where adding chunks of animation helped students' understanding of the materials, the addition of sounds did not help students' learning. We interpret this failure as due to the low informational content of the sounds. However, the addition of environmental sounds did not hinder learning. Consistent with past studies on multimedia learning, adding relevant sounds to a lesson did not hurt students' learning when they were easily associated with the rest of the materials (Moreno \& Mayer, 2000).

\section{Practical Implications}

An important consideration in the design of multimedia presentations aimed at explaining how a scientific system works is whether to provide the verbal explanation through text, audio, or both. An effective technique to promote broader learning with multimedia explanations is to use the auditory and visual modalities simultaneously for verbal information if no other visual material is presented concurrently. The beneficial effects of verbal redundancy, however, are limited to presentations where no other visual information is presented simultaneously to the learner. This limitation is particularly important in light of the increasing use of graphics, on-screen text, and audio in educational software.

\section{References}

Anderson, J. R. (1983). The architecture of cognition. Cambridge, MA: Harvard University Press.

Baddeley, A. (1992, January). Working memory. Science, 255, 556-559.

Baddeley, A. D. (1986). Working memory. Oxford, England: Oxford University Press.

Bobis, J., Sweller, J., \& Cooper, M. (1993). Cognitive load effects in a primary school geometry task. Learning and Instruction, 3, 1-21.

Broadbent, D. E. (1956). Successive responses to simultaneous stimuli. Quarterly Journal of Experimental Psychology, 8, 145-152.

Chandler, P., \& Sweller, J. (1991). Cognitive load theory and the format of instruction. Cognition and Instruction, 8, 293-332.

Chandler, P., \& Sweller, J. (1992). The split-attention effect as a factor in the design of instruction. British Journal of Educational Psychology, 62, 233-246.

Clark, J. M., \& Paivio, A. (1991). Dual coding theory and education. Educational Psychology Review, 3, 149-210.

Colquhoun, W. P. (1975). Evaluation of auditory, visual, and dual-mode displays for prolonged sonar monitoring in repeated sessions. Human Factors, 17, 425-437.

Halpern, J., \& Lanz, A. E. (1974). Learning to utilize information presented over two sensory channels. Perception and Psychophysics, 16, 321-328.

Hede, A. J. (1980). Dichotic and bisensory group effects. Quarterly Journal of Experimental Psychology, 32, 295-306.

Kalyuga, S., Chandler, P., \& Sweller, J. (1998). Levels of expertise and instructional design. Human Factors, 40, 1-17.

Kalyuga, S., Chandler, P., \& Sweller, J. (1999). Managing split-attention and redundancy in multimedia instruction. Applied Cognitive Psychology, 13, 351-371.

Kinchla, R. A. (1974). Detecting target elements in multielement arrays: A confusability model. Perception and Psychophysics, 15, 149-158.

Kobus, D. A., Russotti, J., Schlichting, C., Haskell, G., Carpenter, S., \&
Wojtowicz, J. (1986). Multimodal detection and recognition performance of sonar operators. Human Factors, 28, 23-29.

Lewandowski, L. J., \& Kobus, D. A. (1989). Bimodal information processing in sonar performance. Human Performance, 2, 73-84.

Lewandowski, L. J., \& Kobus, D. A. (1993). The effects of redundancy in bimodal word processing. Human Performance, 6, 229-239.

Macromedia. (1997). Director 5.0 [Computer program]. San Francisco: Author.

Martin, M. (1980). Attention to words in different modalities: Four-channel presentation with physical and semantic selection. Acta Psychologica, 44, 99-115.

Mayer, R. E. (1997). Multimedia learning: Are we asking the right questions? Educational Psychologist, 32, 1-19.

Mayer, R. E., \& Gallini, J. K. (1990). When is an illustration worth ten thousand words? Journal of Educational Psychology, 82, 715-726.

Mayer, R. E., \& Moreno, R. (1998). A split-attention effect in multimedia learning: Evidence for dual processing systems in working memory. Journal of Educational Psychology, 90, 312-320.

Mayer, R. E., \& Sims, V. K. (1994). For whom is a picture worth a thousand words? Extensions of a dual-coding theory of multimedia learning. Journal of Educational Psychology, 86, 389-401.

Montali, J., \& Lewandowski, L. (1996). Bimodal reading: Benefits of a talking computer for average and less skilled readers. Journal of Learning Dissabilities, 29, 271-279.

Moreno, R., \& Mayer, R. E. (1999a). Cognitive principles of multimedia learning: The role of modality and contiguity. Journal of Educational Psychology, 91, 358-368.

Moreno, R., \& Mayer, R. E. (1999b). Designing multimedia presentations with animation: What does the research say? Proceedings of IEEE Multimedia Systems 1999 (pp. 720-725). Florence, Italy: IEEE Press.

Moreno, R., \& Mayer, R. E. (1999c). Visual presentations in multimedia learning: Conditions that overload visual working memory. In D. P. Huijsmans \& A. W. M. Smeulders (Eds.), Lecture notes in computer science: Visual information and information systems (pp. 793-800). Berlin: Springer Verlag.

Moreno, R., \& Mayer, R. E. (2000). A coherence effect in multimedia learning: The case for minimizing irrelevant sounds in the design of multimedia instructional messages. Journal of Educational Psychology, 97, 117-125.

Mousavi, S. Y., Low, R., \& Sweller, J. (1995). Reducing cognitive load by mixing auditory and visual presentation modes. Journal of Educational Psychology, 87, 319-334.

Nickerson, R. (1973). Intersensory facilitation of reaction time: Energy summation or preparation enhancement. Psychological Review, 80, 489-509.

Paivio, A. (1986). Mental representation: A dual coding approach. Oxford, England: Oxford University Press.

Penney, C. G. (1989). Modality effects and the structure of short-term verbal memory. Memory and Cognition, 17, 398-422.

Sweller, J. (1988). Cognitive load during problem solving: Effects on learning. Cognitive Science, 12, 257-285.

Sweller, J. (1989). Cognitive technology: Some procedures for facilitating learning and problem solving in mathematics and science. Journal of Educational Psychology, 81, 457-466.

Treisman, A. M., \& Davies, A. (1973). Divided attention to eye and ear. In S. Kornblum (Ed.), Attention and performance IV (pp. 101-117). New York: Academic Press.

Received January 2, 2001

Revision received May 14, 2001

Accepted May 14, 2001 